\title{
Three-dimensional effects in polarization signatures as observed from precipitating clouds by low frequency ground-based microwave radiometers
}

\author{
A. Battaglia ${ }^{1}$, C. Simmer ${ }^{1}$, and H. Czekala ${ }^{2}$ \\ ${ }^{1}$ Meteorological Institute, University of Bonn, Bonn, Germany \\ ${ }^{2}$ RPG Radiometer Physics GmbH, Meckenheim, Germany
}

Received: 3 April 2006 - Published in Atmos. Chem. Phys. Discuss.: 27 June 2006

Revised: 30 August 2006 - Accepted: 22 September 2006 - Published: 27 September 2006

\begin{abstract}
Consistent negative polarization differences (i.e. differences between the vertical and the horizontal brightness temperature) are observed when looking at precipitating systems by ground-based radiometers at slant angles. These signatures can be partially explained by onedimensional radiative transfer computations that include oriented non-spherical raindrops. However some cases are characterized by polarization values that exceed differences expected from one-dimensional radiative transfer.
\end{abstract}

A three-dimensional fully polarized Monte Carlo model has been used to evaluate the impact of the horizontal finiteness of rain shafts with different rain rates at 10, 19, and $30 \mathrm{GHz}$. The results show that because of the reduced slant optical thickness in finite clouds, the polarization signal can strongly differ from its one-dimensional counterpart. At the higher frequencies and when the radiometer is positioned underneath the cloud, significantly higher negative values for the polarization are found which are also consistent with some observations. When the observation point is located outside of the precipitating cloud, typical polarization patterns (with troughs and peaks) as a function of the observation angle are predicted. An approximate 1-D slant path radiative transfer model is considered as well and results are compared with the full 3-D simulations to investigate whether or not three-dimensional effects can be explained by geometry effects alone. The study has strong relevance for low-frequency passive microwave polarimetric studies.

Correspondence to: A. Battaglia

(batta@uni-bonn.de)

\section{Introduction}

Microwave polarization signatures related to cloud systems and observed by passive ground-based or space-borne radiometers have been reported by several authors (Heymsfield and Fulton, 1994; Spencer et al., 1989; Prigent et al., 2001, 2005; Kutuza et al., 1998; Czekala et al., 2001a; Troitsky et al., 2003; Liu, 2004). Since no other polarization sources (e.g. polarized emission/scattering by surfaces) are in place these signatures are related to the interaction of radiation with the cloud constituents and can be attributed to non-spherical hydrometeors (like raindrops or ice crystals) with preferred orientations. Many theoretical studies (for a detailed review and references see Haferman, 2000) demonstrated that the interaction with atmospheric constituents changes the polarization state of radiation. Even for locally isotropic radiation sources (like spherical water/ice particles), multiple scattering produces some amount of polarization by itself (e.g. see Liu and Simmer, 1996). However the presence of dichroic media makes polarization signatures more likely and causes a much wider variety of features (e.g. Czekala, 1998; Czekala and Simmer, 1998, 2002; Evans and Stephens, 1995; Evans et al., 1998; Battaglia and Simmer, 2006 ${ }^{1}$.

Polarimetric passive microwave measurements have been rarely exploited to quantitatively retrieve properties of the hydrometeors contained in the field of view. On the other hand active sensors with polarization diversity considerably ameliorate hydrometeor retrievals. E.g., rainfall estimates receive a considerable improvement when dual-polarized radar measurements are performed (see Sect. 7 in Bringi and Chan-

\footnotetext{
${ }^{1}$ Battaglia, A. and Simmer, C.: Explaining the polarization signal from rain dichroic media, J. Quant. Spectrosc. Radiat. Transfer, submitted, 2006.
}

Published by Copernicus GmbH on behalf of the European Geosciences Union. 


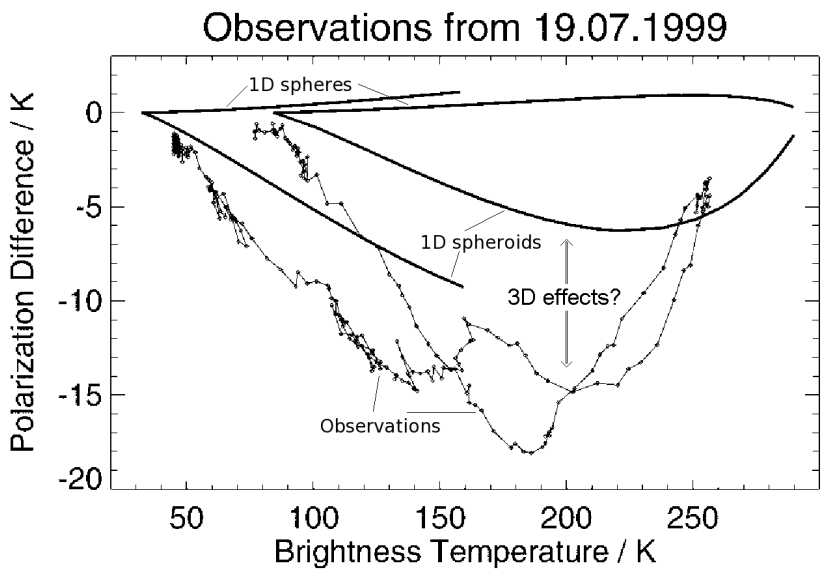

Fig. 1. Comparison of 1-D model results for spherical (upper continuous lines) and non spherical raindrops (lower continuous lines) and observations (points) for a warm rain event, occurred on 19 July 1999. Adapted from Czekala et al. (2001a).

drasekar, 2001 and references therein). The basis for this information is the well defined equilibrium shape of raindrops and their orientation distribution in absence of turbulence and wind shear. Based on the same physical foundation, Czekala et al. (2001b) proposed to discriminate cloud and rain liquid water path by ground-based polarized microwave radiometry. Although ground-based microwave radiometry is a fairly established technique to retrieve the verticallyintegrated liquid water path (LWP), water vapor profiles, and temperature profiles (e.g. Janssen, 1996), current LWP retrievals (not using polarized observations) are limited in accuracy by the presence of drizzle and rain, which introduces a substantial change in the proportionality between water mass and brightness temperature $\left(T_{B}\right)$. Cloud droplets have a different $T_{B}$ per water mass ratio than larger raindrops (Rayleigh scattering versus Mie scattering). Since larger drops exhibit a polarization signature in the downwelling microwave radiation, such ambiguities can potentially be significantly reduced with polarized observations. This idea has been never fully tested by field measurements. Nevertheless, ground based observations (e.g. Kutuza et al., 1998; Czekala et al., 2001a) have confirmed distinctive signatures (i.e. the presence of strong negative polarizations) in low microwave frequencies downwelling radiation coming from raining clouds. An example of the mean polarisation difference $P D=T_{B}^{V}-T_{B}^{H}$ (with corresponding standard deviation) ground-based observations performed with a $19.2 \mathrm{GHz}$ dual polarization radiometer at $30^{\circ}$ elevation angle in Southern Germany for a total of 513 observation days and sorted according to the $T_{B}$ values can be found in Fig. 6 in Czekala et al. (2001a). $P D$ s show a typical negative signal first decreasing with increasing $T_{B}$, then saturating around $200 \mathrm{~K}$ and finally increasing towards zero for $T_{B}>220 \mathrm{~K}$. This general behavior of the $P D$ s can be explained by radiative trans- fer computations (e.g. Czekala and Simmer, 1998; Czekala et al., 1999) which involve a 1-D raining cloud setup containing perfectly aligned raindrops. Spherical raindrops can produce only positive signals in this same setup.

However, in this same observational dataset, Czekala et al. (2001a) noticed the presence of events with very bad agreement between model and observations. For instance, Fig. 1 (which is adapted from Fig. 17 by Czekala et al., 2001a) demonstrates that, for some events, the ground based observations (dotted line) cannot be simulated at all in a 1-D setup. In Fig. 1, the two lower continuous lines represent results obtained with 1-D non-spherical rain layers of different thickness; the warmest profile corresponds to the thickest layer, which has a temperature profile with higher temperature close to the ground. The different points in each curve corresponds to different rain rates ( $R R$ hereafter). This would suggest that the observational points correspond to the temporal development of the rain shower with its corresponding intensity evolution.

When separating each single shower event the $\left(T_{B}, P D\right)$ measurements generally present a concave shape (or part of it) similar to that reproduced with 1-D radiative transfer simulations performed with different $R R$. However the observed shapes differ from the 1-D simulations for different features.

1. Amplitude of the minimum: observations are characterized by the presence of strong negative $P D$ s with signals down to $-18 \mathrm{~K}$ which cannot be reproduced at all in a 1-D scenario.

2. Position of the minimum: this is located at lower $T_{B} \mathrm{~s}$ in the observation curve than in the simulated one.

3. Slope of the ascending and descending part: for the observations the slope is much more negative/positive in the descending/ascending part.

Coincidental observations indicate high $R R$ for this event; this suggests a convective precipitation type, which is typically characterized by small horizontal scales. Hence Czekala et al. (2001a) conclude that 3-D effects are very likely to be present in this and in other similar situations, but no studies have been performed to support this idea.

It is the main goal of this paper to investigate whether or not the pattern shown in Fig. 1 can be explained in a setup which includes both dicroic media and 3-D configurations. More generally the paper aims (a) at understanding how the 3 -D structure can affect both the $T_{B}$ s and the $P D \mathrm{~s}$ at all frequencies (10-19-30 GHz) and in all viewing configurations that are likely to be used for rain/cloud liquid water discrimination and (b) at explaining characteristic patterns and signatures caused by 3-D rainy structures. The analysis is extended to all polarization channels, including the third and the fourth Stokes components.

To achieve these goals, the 3-D vector radiative transfer equation (VRTE hereafter) is solved for 3-D scenarios 
involving non-spherical raindrops by a backward-forward methodology, briefly described in Sect. 2. The model is applied to simple cloud box scenarios described in Sect. 3. Results presented in Sect. 4 are inter-compared with 1-D slant path (SP hereafter) approximations in Sect. 5. Section 6 revisits 3-D results in the light of the weaknesses of 1-D modeling illustrated in Fig. 1. Finally conclusions are drawn in Sect. 7.

\section{Method of solution of the vector radiative transfer equation: backward-forward Monte Carlo}

The VRTE (for details see Haferman, 2000) represents the basic equation to describe the interaction between radiation and the atmospheric constituents. In its general form it can be solved with many different methodologies, a review of which is provided e.g. by Mätzler (2006). Different techniques have been developed to numerically treat the radiative transfer equation for the full Stokes vector in a 3-D environment in the presence of dichroic media (Haferman et al., 1993; Kutuza et al., 1998; Davis et al., 2005; Battaglia and Mantovani, 2005). A recent intercomparison study has demonstrated that because of its lower computational cost the backward-forward Monte Carlo technique (Liu et al., 1996) based on importance sampling (Davis et al., 2005) represents the most efficient way to face passive microwave radiative transfer problems related to optically thick 3-D structured clouds including non spherical preferentially oriented hydrometeors. Therefore, the VRTE has been solved by a forward-backward Monte Carlo scheme. In this work the algorithm is a recoding of the scheme presented by Davis et al. (2005) in a Cartesian grid without using the concept of a "cloud box". The cloud structure is embedded in a background atmosphere like in Battaglia and Mantovani (2005) while the surface is described by either a Fresnel or a Lambertian model. The interaction with the surface is treated in a way similar to an interaction with an atmospheric constituent (Eqs. 9-13 in Davis et al., 2005) by applying importance sampling. In the backward segment the probability of scattering by the surface is evaluated for non-polarized radiation, $p_{\text {unpol. }}$. Note that, for Fresnel surfaces, this is not the true probability since in this case the true probability depends on the polarization state of the incident radiation. In order to take this into account, a biasing technique is applied in the forward segment:

- in case of a scattering event, the Stokes vector impinging at the surface is normalized by $p_{\text {unpol }}$ and then multiplied with the bidirectional reflection matrix of the surface (see Eqs. 35-37 in Haferman, 2000);

- in case of an absorption event, a photon is emitted from the surface with an emission vector proper to the surface type (see Eqs. 36-40 in Haferman, 2000) normalized by the factor $\left(1-p_{\text {unpol }}\right)$.

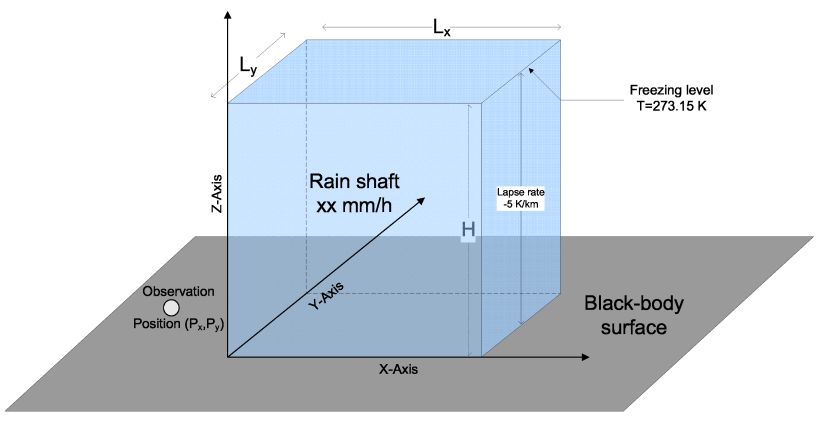

Fig. 2. Schematic for the rain cloud simulation. Radiances have been computed at observation points located at the location $\left(P_{x}, P_{y}\right)$. The blue-shaded area contains the rain system. Non shaded areas contain only atmospheric gases.

The algorithm has been recently developed in the frame of a radiative transfer intercomparison study and validated with other Monte Carlo schemes, Battaglia et al. (2006).

\section{Box type cloud model}

In order to quantify the effects of the 3-D structure of a raining cloud on the radiation field sensed by a polarimetric ground-based radiometer, the backward-forward method is applied to a box-type cloud model, as illustrated in Fig. 2. $L_{x}$ and $L_{y}$ are the horizontal dimensions of the rain shaft while $H$ is its height. The three numbers $\left(H, L_{x}, L_{y}\right)$ expressed in km define a specific cloud configuration. For instance, configuration " 422 " refers to a cloud box with a $4 \mathrm{~km}$ height and with a square horizontal dimension of $2 \mathrm{~km}$. The cloud box contains horizontally oriented raindrops, modeled as oblate spheroids. The axial ratios (lower than 1) are parameterized according to Andsager et al. (1999) as a function of equivalent spherical raindrop diameter $\mathrm{D}$, while the drop size distribution is an exponential Marshall and Palmer with different rainfall rates. The single scattering properties (i.e. the extinction and the phase matrix, and the emission vector) are computed according to Mishchenko (2000). The surface is assumed to be Lambertian with emissivity equal to 1 . The atmosphere is supposed to be water vapour saturated with a linear temperature lapse rate of $-5 \mathrm{~K} / \mathrm{km}$; the temperature of the top of the rain layer, $T_{\text {top }}$, coincides with the freezing layer. Cosmic radiation impinges at $T_{c}=2.7 \mathrm{~K}$ at the top of the atmosphere.

The downwelling Stokes vectors at the ground are computed at different positions relative to the rain shaft for 21 zenith viewing angles with cosines sampled between -1 and 0 with step 0.05 . The radiances are simulated as sensed by a radiometer with an infinite angular resolution either located underneath the cloud looking upward or looking at the side of the cloud from outside of the rain shaft (this second option is the one depicted in Fig. 2). The position of the observation 
point is individuated by the coordinates $\left(P_{x}, P_{y}\right)$, as referred to the coordinate axis plotted in Fig. 2.

Although the scenario is very simple, it follows previous similar 3-D modeling (e.g. Weinman and Davies, 1978; Kummerow and Weinman, 1988; Liu et al., 1996) and it is the natural extension of the 1-D scenarios proposed in Czekala et al. (1999), which are the basis to explain the experimental polarimetric measurements observed at the bottom of a raining layer in Czekala et al. (2001a). No ice phase and no melting hydrometeors have been considered. While ice particles have been found to have a small effect on the downwelling signal (Czekala and Simmer, 2002), the melting layer will certainly produce an enhancement of the $T_{B} \mathrm{~s}$, due to the extinction peak and the increase in the optical thickness, a typical signature at low microwave frequencies (Battaglia et al., 2003 and references therein). Practically, the cloud will appear thicker than it actually is. As regards to the $P D$ signal, although the evolution of the axial ratio of a melting particles is not straightforward (like shown in Raynaud et al., 2000), certainly the bright band will correspond to a peak in the differential attenuation as well, which is more marked at lower frequencies (e.g. panel (b) in Figs. 6-7 by Zhang et al., 1996). Since events affected by 3-D effects are supposed to have a more convective nature (thus no bright band) the detailed evaluation of this effect remains out of the scope of this work.

\section{Results}

As a first example a " 444 " rain shaft with $R R=10 \mathrm{~mm} / \mathrm{h}$ is considered. Results for $I$ and $Q$ at the three frequencies investigated and at different observation points are plotted in Fig. 3. Each symbol curve of Fig. 3 represents a different observation point as indicated in the legend. While in the $I$ panels two additional curves have been added (the 1-D (continuous) and the clear sky (dashed) solution), in the $Q$ panels the clear sky solution (always zero) has been omitted.

Depending on the viewing angle and to the observation point $T_{B}$ s assume intermediate values between 1-D and clear sky $T_{B}$ s. Obviously when looking at the cloud from the outside $\left(P_{x}<0\right)$ for zenith angles $\theta_{z}$ satisfying $H \tan \left(\theta_{z}\right) \leq\left|P_{\mathrm{x}}\right|$ (e.g. for $\mu<-0.93$ or $\mu<-0.75$ for the square and the cross line, respectively in Fig. 3) the solution coincides with the clear sky solution. Conversely when looking at the cloud from the inside $\left(P_{x}>0\right)$ for zenith angles satisfying $H \tan \left(\theta_{z}\right) \leq L_{x}-P_{x}$ (e.g. for $\mu<-0.99$ or $\mu<-0.94$ or $\mu<-0.75$ for the triangle, the dash-dotted and the diamond line respectively in Fig. 3) the solution is approximately equal to the 1-D solution. For these observation geometries, 3-D results are generally colder than the 1-D approximation because of photons leaking from the side of the cloud (Kummerow and Weinman, 1988; Roberti et al., 1994; Liu et al., 1996; Bauer et al., 1998): while at the lowest frequency this effect is practically undetectable it becomes more and more visible at higher frequencies (e.g. $2.2 \mathrm{~K}$ at $19.4 \mathrm{GHz}$ and $8 \mathrm{~K}$ at $30.0 \mathrm{GHz}$ at nadir) because of the larger single scattering albedo ( $S S A$ hereafter). The intensities are largely determined by the cloud sensed optical thickness (while the clear sky gas absorption optical thickness remains the same in all configurations) with higher $T_{B}$ s produced by higher cloud sensed optical thicknesses. The larger sensed optical thickness in 1-D configurations explains why $T_{B}[1-D] \geq T_{B}[3-D]$. Close to grazing angles all the intensities observed from outside (square and cross lines in Fig. 3) converge approximately to the same solution, since the same cloud optical thickness (that one corresponding to a cloud thickness $L_{x}$ at horizontal directions) is sensed. When looking at the rain shaft from underneath the cloud (triangle, dash-dotted and diamond lines in Fig. 3) $T_{B}$ s constantly decrease when moving from $P_{x}=0$ to $P_{x}=L_{x}$ because of the reduction in the sensed optical thickness.

For the polarization fields, the results of 3-D scenarios are quite different from those obtained from a plane-parallel cloud as well. However, for these quantities results are not confined between the 1-D approximation and the clear sky fields (zero polarization) and a great variety of patterns is found depending on the viewing position, the sensed optical thickness and the scattering regime. Battaglia and Simmer $(2006)^{1}$ showed that, at low microwave frequencies (like considered here), a crucial role is played by the 0 -order scattering term (hereafter indicated by an apex [0]), which in the limit of high and small sensed optical thicknesses in a 1-D geometry becomes:

$$
\begin{aligned}
& \lim _{\tau_{s l}^{\gg 1}} P D^{[0]}(\mu)=-T_{\text {bot }} \underbrace{\left[\varpi_{V}(\mu)-\varpi_{H}(\mu)\right]}_{\text {term A }} \\
& \lim _{\tau_{s l}^{\ll} 1} P D^{[0]}(\mu)=\underbrace{T_{c}\left(\tau_{s l}^{H}(\mu)-\tau_{s l}^{V}(\mu)\right)}_{\text {term B }}+\frac{T_{\text {top }}+T_{\text {bot }}}{2} \\
& \underbrace{\left(\tau_{s l}^{V}(\mu)\left[1-\varpi_{V}(\mu)\right]-\tau_{s l}^{H}(\mu)\left[1-\varpi_{H}(\mu)\right]\right)}_{\text {term } \mathrm{C}}
\end{aligned}
$$

respectively, where $T_{\text {top }}$ and $T_{\text {bot }}$ are the brightness temperature at the top and at the bottom of the rain layer, $\varpi_{V}$ and $\varpi_{H}\left(\tau_{s l}^{V}\right.$ and $\left.\tau_{s l}^{H}\right)$ are the $S S A$ s (slanted optical thicknesses of the rain layer) for vertically and horizontally polarized radiation in the viewing direction. When reverting to a 3-D configuration expressions similar to Eqs. (1-2) apply. We only have to replace $T_{\text {top }}$ with the temperature at the highest point in the interception region between the SP and the cloud and $T_{C}$ with the downwelling $T_{B}$ (induced by gas emission plus cosmic background) impinging at that point in the viewing direction. Especially at grazing angles this quantity can be much higher than $T_{c}=2.7 \mathrm{~K}$; if so "term B" on the right hand side of Eq. (2) will tend to cancel the effect of "term C". Moreover in 3-D scenarios an additional $P D$ reduction 

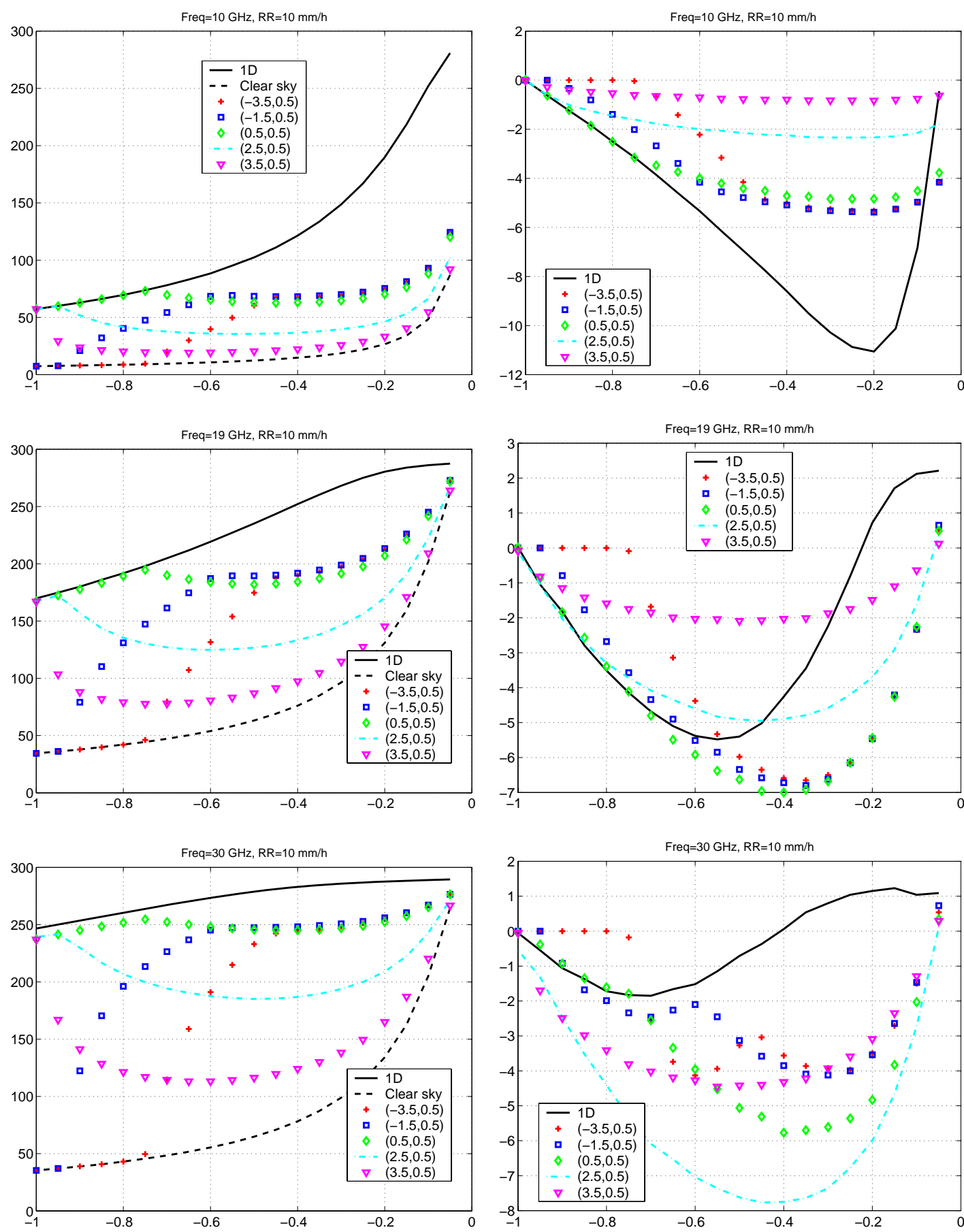

Fig. 3. Intensity $I$ (left panels) and $P D$ s (right panels) evaluated at different observation positions $\left(P_{x}, P_{y}\right)$ (see Fig. 2 for the reference frame) as indicated in the legend. Top, centre and bottom row correspond to $10.7 \mathrm{GHz}, 19.4 \mathrm{GHz}$ and $30.0 \mathrm{GHz}$, respectively. A " 444 " rain shaft (see text for explanation) with $R R=10 \mathrm{~mm} / \mathrm{h}$ is considered.

is caused by the gas layer present between the observation point and the rain shaft in configurations with $L_{x}<0$.
In general, for horizontally aligned particles, $P D^{[0]}$ tends to be positive/negative for thick/thin layers: in Eq. (2) the first term is generally negligible ( $T_{c}$ is small), while both "term A" and "term C" in Eq. (1) and Eq. (2) take negative values. As a result, positive down-welling $P D$ s are induced 


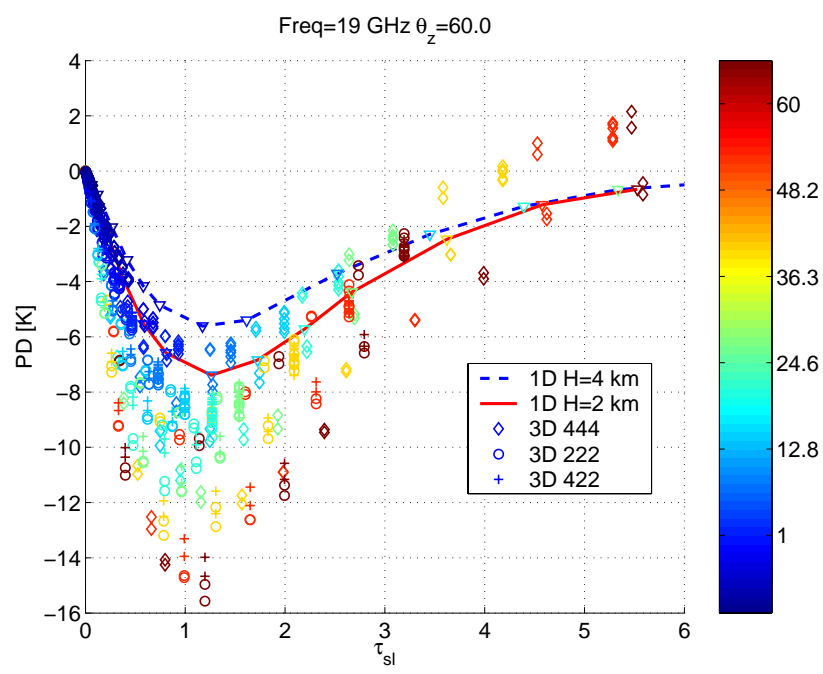

Fig. 4. Scatter plot of the slant optical thickness versus $P D$ s at $19.4 \mathrm{GHz}$ and for $\mu=-0.5$. The color of the scatter-points is modulated by the rain-rate of the cloud box.

by propagation effects when thick layers are sensed while negative $P D$ s (proportional to the slant optical thickness) are induced by emission when thin layers are sensed. To better demonstrate this property, Fig. 4 shows $19.4 \mathrm{GHz} P D$ s as a function of the slant optical thickness for a 1-D rain layer of thickness equal to 2 and to $4 \mathrm{~km}$ (continuous and dashed lines, respectively). Since the zenith angle is fixed at $60^{\circ}$ ( $\mu=-0.5$ ) the optical thickness is increased by varying the $R R$ from 0 to $60 \mathrm{~mm} / \mathrm{h}$. At small optical thickness $P D$ s are negative and decrease linearly with $\tau_{s l}=0.5\left(\tau_{s l}^{V}+\tau_{s l}^{H}\right)$, they reach a minimum around $\tau_{s l} \approx 1.2$, then they increase towards values close to zero corresponding to high values of $\tau_{s l}$, i.e. to high $R R$ s. Note that for the two 1-D curves $P D \rightarrow 0 \mathrm{~K}$ and not to positive values: at high $R R \mathrm{~s} S S A \mathrm{~s}$ are not negligible so that higher order scattering terms tend to mask the $P D^{[0]}$ signatures (Battaglia and Simmer, 2006 ${ }^{1}$ ), which would produce a positive signal. Similar patterns (not shown) have been found at different viewing angles with the position of the minimum always around $\tau_{s l} \approx 1$ but with larger negative values for the minima at more slant viewing angles; moreover, at viewing angles greater than $70^{\circ}$ a relative maximum with slightly positive values appears around $\tau_{s l} \approx 6$. After that $P D$ s decrease toward zero values. At grazing angles, higher $\tau_{s l}$ are reached at lower $R R \mathrm{~s}$; therefore $S S A \mathrm{~s}$ are still low and the $P D^{[0]}$ signature associated with Eq. (1) can show up. In Fig. 4 the continuous curve corresponding to a $2 \mathrm{~km}$ thick rain layer is characterized by a more negative minimum $(-7.3 \mathrm{~K})$ than the $4 \mathrm{~km}$-thick rain layer $(-6.1 \mathrm{~K})$. In effect, the condition $\tau_{s l} \approx 1.2$ is satisfied at $R R$ s equal to $15 \mathrm{~mm} / \mathrm{h}$ and to $8 \mathrm{~mm} / \mathrm{h}$, respectively; thus, since higher $R R \mathrm{~s}$ imply larger and more elongated raindrops, higher $P D$ s are found.

In Fig. 4 results from 3-D scenarios "444" (diamond symbols), "222" (circle symbols) and "422" (cross symbols) are

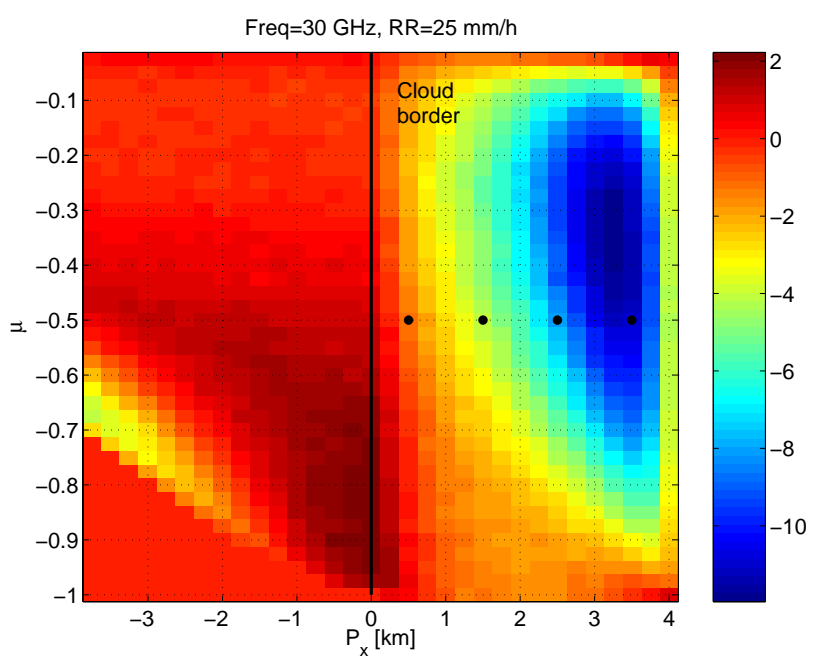

Fig. 5. Variation of the $P D$ field at $30.0 \mathrm{GHz}$ as a function of the $P_{x}$ position and of the cosine of the viewing angle at $P_{y}=0.5 \mathrm{~km}$ for configuration " 444 " with $R R=25 \mathrm{~mm} / \mathrm{h}$.

superimposed. Scatter points correspond to observation positions with $P_{x}=-3.5,-2.5, \ldots 3.5$ and $P_{y}=0.5,1.5$ in the "444" configuration and with $P_{x}=-3.75,-3.25, \ldots 1.75$ and $P_{y}=0.25,0.75$ in the " 422 " and " 222 " configurations. Two main features are evident in the 3-D scenarios: more negative values are found in the region at small $\tau_{s l}$ (and for $\tau_{s l} \approx 1.2$ around the minimum) while positive $P D$ results are obtained in the region of larger $\tau_{s l}$. For the analyzed 3-D configurations with a fixed rain shaft height $H$, the same optical thickness can be achieved with different $R R \mathrm{~s}$ : as before, at small $\tau_{s l}$, highest $R R$ s produce more negative $P D \mathrm{~s}$. When reverting to the region at high $\tau_{s l}$ positive values up to $2.4 \mathrm{~K}$ are reached for viewing positions with $P_{x}<0$. This is possible because, in contrast to what happens for the 1-D case, the contributions from higher order of scattering do not cancel the zero-order of scattering contribution.

In the light of these considerations some features of the panels in Fig. 3 can be better understood. At the lowest frequency $10.4 \mathrm{GHz}$ (see top right panel in Fig. 3) emission/absorption processes are dominant so that the $P D$ s are essentially determined by the term $P D^{[0]}$ : 3-D effects can be interpreted here as pure geometrical ones, i.e. they can be evaluated by taking into account the geometrical variation of the sensed optical thickness. In this case, in the 1-D approximation, $\tau_{s l}<1$ for all $\mu<-0.1$, so that the approximation (2) can be used. A fortiori Eq. (2) can be applied for all viewing positions in the 3-D scenario as well. Since in this regime the $P D$ s are proportional to the sensed optical thickness a reduction (compared to the 1-D approximation) of the $P D \mathrm{~s}$ is found in all 3-D configurations, which is linearly proportional to the reduction in $\tau_{s l}$. When increasing the frequency at constant $R R$ (i.e. moving from the top to the bottom in Fig. 3) the regime of small slant optical thicknesses is con- 
fined toward zenith angles closer to nadir. Obviously, in the 3-D configurations, due to the reduction of the slant optical thickness, this region can actually extend to higher $\mu$ values. Although higher scattering orders become increasingly more important at higher frequencies the 0 -order of scattering term still strongly affects the total $P D$ signal. For instance, the signature of this term can be seen in the typical $P D$ patterns with troughs and peaks for observation points outside of the cloud (square and cross lines in bottom right panel of Fig. 3). As a rule of thumb, at the same viewing angle, more negative $P D$ s are expected for those viewing positions which have slant optical thickness closer to 1.0. As an order of magnitude, the optical thickness of a $4 \mathrm{~km}$-thick $10 \mathrm{~mm} / \mathrm{h}$ layer is approximately $0.2,0.8$ and 2.1 at the three frequencies under investigation, respectively.

Figure 5 presents a cross section of the $P D$ field at $P_{y}=1.5 \mathrm{~km}$ as a function of the position $P_{x}$ and of the viewing angle $\mu$ for a $R R=25 \mathrm{~mm} / \mathrm{h}$ at $30.0 \mathrm{GHz}$. Both strongly negative and slightly positive $P D$ s can be found; on the contrary, for this case, the 1-D approximation predicts $P D$ s always very close to zero with values between -0.12 at $\mu=-0.8$ and 0.1 at grazing angles. In effect, in the 1-D configuration the radiometer always senses an optical thickness larger than 5 while in the 3-D scenario the sensed optical thickness can be much smaller. This is particularly true in the region where $P_{x} \geq 3 \mathrm{~km}$ with viewing angles far from nadir. Note that the largest variation in $P D$ s is met when moving the observation positions underneath the cloud; when looking the cloud from outside differences are less pronounced especially at low elevation angles when the same cloud slant optical thickness is intercepted by the viewing beam.

\section{3-D effects: intercomparison with a 1-D slant path ap- proximation}

3-D effects are generally regarded as caused by geometrical and by scattering factors (Battaglia et al., 2005 and reference therein). When scenarios with weak scattering are considered the leakages from the warm side of the cloud can be accounted for by 1-D SP approximation (Liu et al., 1996; Bauer et al., 1998; Roberti and Kummerow, 1999). On the other hand for scattering scenarios, 3-D effects have to be evaluated on a case by case basis.

1-D SP approximation-based calculations have been performed with the fast RT4 code (Evans and Stephens, 1991) and compared with 3-D computations. In Fig. 6 the differences between the two methodologies are drawn for the same cases illustrated in Fig. 3. Results are practically the same where clear sky conditions are met (e.g. at nadir for all positions with $\left.P_{x}<0\right)$. Departures are quite small at $10 \mathrm{GHz}$ with $T_{B}$ s and $P D$ s always within $0.5 \mathrm{~K}$ and $0.05 \mathrm{~K}$, respectively except at close to grazing angles $(\mu \geq-0.15)$. This is expected since at this $R R$ the $S S A$ is lower than 0.07 while $k_{\mathrm{ext}} \approx 0.05 \mathrm{~km}^{-1}$. Thus the predominant term affecting the to- tal signal is the zero order of scattering, which is perfectly accounted for by the SP approximation. But this methodology takes only approximately into account successive orders of scattering. In effect, these contributions depend on the radiation field impinging at each point of the SP from any direction, so that it cannot be correctly evaluated in a 1-D SP approximation. On the other hand strong departures are found at $30.0 \mathrm{GHz}\left(S S A \approx 0.32, k_{\mathrm{ext}} \approx 0.5 \mathrm{~km}^{-1}\right)$ where differences in $T_{B} \mathrm{~s}(P D \mathrm{~s})$ can be as high as $15 \mathrm{~K}(5 \mathrm{~K})$ at grazing views (bottom panels of Fig. 6). Because of the leakages from the side of the clouds $T_{B}$ s results in the 1-D SP approximation have larger discrepancies $(\approx 10 \mathrm{~K})$ at nadir as well. These large discrepancies are certainly attributable to the deficiencies of the 1-D SP model in computing terms with orders of scattering $\geq 1$. These simple examples demonstrate that the 1-D SP approximation does not provide acceptable results except when low frequency and small $R R$ s are considered.

Another way to show the necessity of a full 3-D radiative transfer model is to evaluate the radiation field when moving in the Y-direction at a fixed $L_{x}$ within the cloud (see Fig. 2). In Fig. 7 the results of a Y-cross section are depicted in correspondence to the four positions individuated by the star-symbols in Fig. 5. A 1-D SP model obviously predicts no variation of the radiation field when moving along the $\mathrm{Y}$ direction because the SP is the same for all these viewing positions (all located underneath the cloud). Vice versa Fig. 7 shows that, for a rain shaft at $25 \mathrm{~mm} / \mathrm{h}$ at $30 \mathrm{GHz}(S S A \approx 0.4$, $k_{\text {ext }} \approx 1.5 \mathrm{~km}^{-1}$ ) departures as large as $3 \mathrm{~K}$ and $15 \mathrm{~K}$ can be found in $P D$ and $T_{B}$ patterns, respectively.

Finally, in the 3-D simulations some peculiar aspects (already noted in Battaglia et al., 2006) are present: $P D$ s different from zero can be found at nadir (see bottom right panel in Fig. 3 and upper part in the panel of Fig. 5) and the $U$ and $V$ channels are generally different from zero. While the fourth Stokes parameter remains always very low the $U$ parameter shows a signal which would be certainly detectable. An example of the $U$ field is shown in Fig. 8 where values as high as $1.8 \mathrm{~K}$ are reached. Note that $U$ values different from zero are generated just because of the 3-D structure (i.e. the horizontal in-homogeneity) of the simulation. It is worthwhile reminding the reader that raindrops perfectly aligned in the horizontal plane (i.e. with zero drop canting angle) are considered in this study. Kutuza et al. (1998) analysed the radiometric response of $1-\mathrm{D}$ rain clouds containing canted raindrops at frequencies in the range $6-35 \mathrm{GHz}$. They concluded that "the first azimuthal harmonic of the third Stokes parameter for emission is proportional to the average canting angle of the oblate raindrops" and showed that the magnitude of $U$ reaches maximum values of the order of $2-3 \mathrm{~K}$ for realistic distributions of the drop canting angle (see their Fig. 9). The same order of magnitude has been found by our computations as summarized by the right panel in Fig. 8. Therefore, a general understanding of the behavior of the $U$ signal generated by raining clouds should include both $3-\mathrm{D}$ and canting effects. 

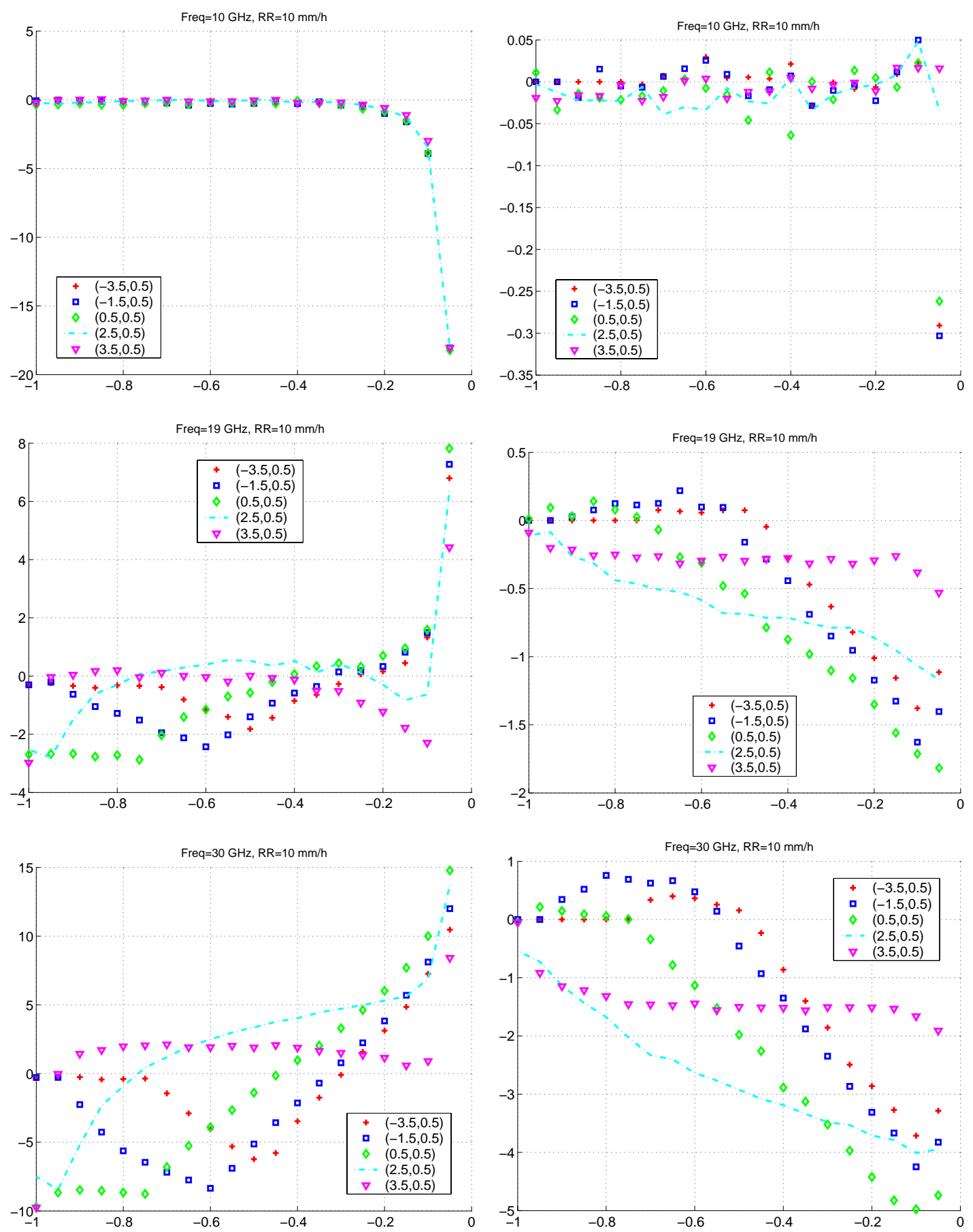

Fig. 6. Difference between the 3-D computation and the 1-D SP approximation in correspondence to the same panels of Fig. 3.

\section{The $T_{B}-P D$ relationship}

In order to better compare our simulations with the measurements in Fig. 1 a scatter plots obtained with three 3-D configurations ("444" (diamonds), "222" (circles), " $422 "$ (crosses)) at an elevation angle equal to $30^{\circ}$ is depicted in Fig. 9. Each point in the scatter plots corre- sponds to a couple $\left(T_{B}, P D\right)$ simulated at a different observation positions (like for Fig. 4) for a rain shaft with $R R=1,2 \ldots, 10,15, \ldots, 30,40,50,60 \mathrm{~mm} / \mathrm{h}$. Its color relates to the $R R$ of the rain shaft (as indicated in the color bar). The continuous and dashed line corresponding to the 1-D solutions with $H=2,4 \mathrm{~km}$ are included for completeness in Fig. 9. Obviously, the 3-D geometry introduces a much 

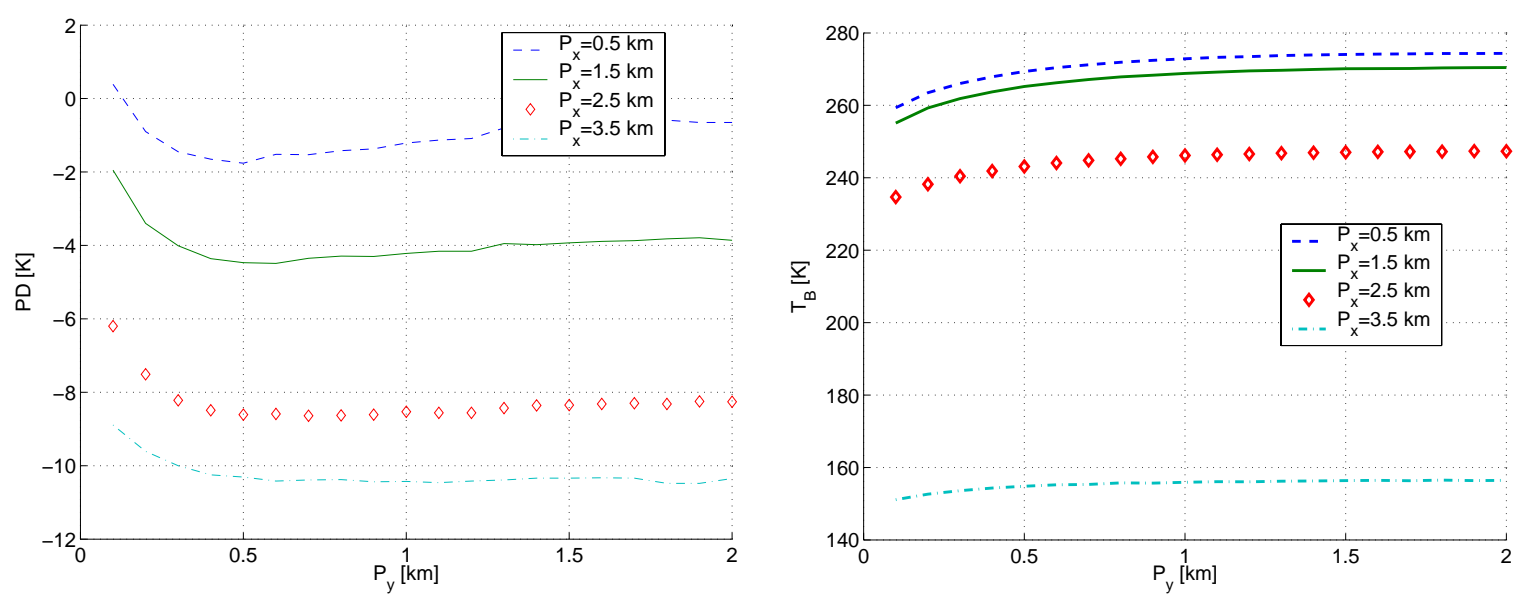

Fig. 7. Variation of the $P D$ (top) and $T_{B}$ field (bottom) at $30.0 \mathrm{GHz}$ for a " 444 " rain shaft with $R R=25 \mathrm{~mm} / \mathrm{h}$ at constant slant optical thickness at $\mu=-0.5$. Different $P_{x}$ corresponds to different slanted optical thicknesses.
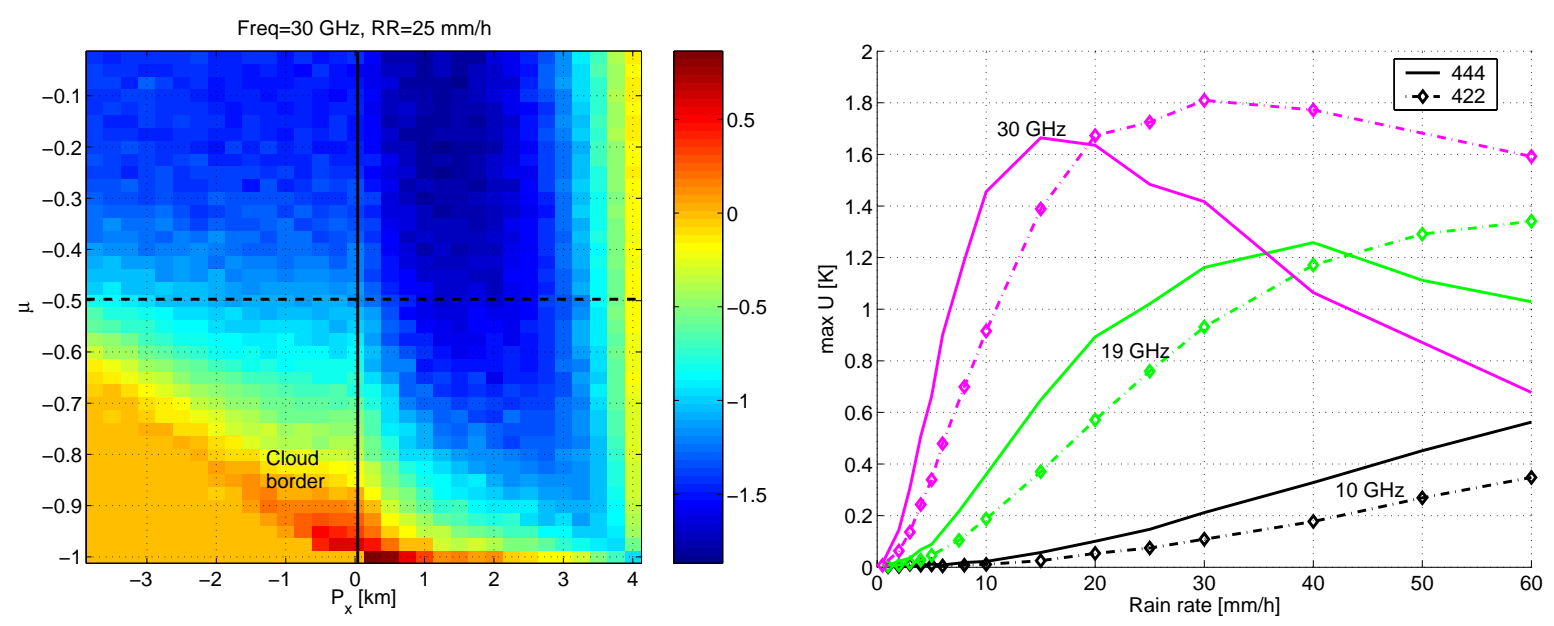

Fig. 8. Left panel: same as Fig. 5 for the $U$ field. Right panel: maximum values assumed by the absolute value of the third Stokes component at $\mu=-0.5$ for different rain rates, $3-\mathrm{D}$ configuration and frequencies.

wider variability of possible combinations of $T_{B} s$ and $P D \mathrm{~s}$. This allows to explain experimental observations like those depicted in Fig. 1 and seems to overcome the weaknesses of the 1-D model listed in Sect. 1.

Another important consideration: while in the frame of a 1-D model with a fixed freezing level and temperature/water vapor profile, the time evolution of the $\left(T_{B}, P D\right)$ observations can be explained only in terms of a variation of the $R R$ of the rain shaft, within 3-D scenarios the same observations can be explained also by a movement (relative to the observation point) of the rain shaft (at fixed $R R$ ). This is demonstrated in the middle and bottom scatter plots of Fig. 9 by the black dash-dotted lines, which represent the different $\left(T_{B}, P D\right)$ couples which can be generated by the same box rain shaft when observed from different positions. At $19.4,30.0 \mathrm{GHz} R R$ s of $10 \mathrm{~mm} / \mathrm{h}, 25 \mathrm{~mm} / \mathrm{h}$ are selected re- spectively. For each of these curves, points with lower $T_{B} \mathrm{~S}$ generally correspond to observation points underneath the cloud, with nearby cloud boundaries in the viewing direction.

Scatter plots like Fig. 9 do not indicate that 3-D effects produce on average more negative polarization. In effect, the relative distribution of $R R \mathrm{~s}$ has to be taken into consideration. At $10 \mathrm{GHz}$ for $R R \mathrm{~s}$ lower than $10 \mathrm{~mm} / \mathrm{h}$ (which are the most commonly found) 3-D effects tend actually to decrease the amplitude of the $P D$ signal with respect to the 1-D case. On the contrary, at $30.0 \mathrm{GHz}$ a considerable amount of points with very negative polarization is generated by a rain shaft with $R R<15 \mathrm{~mm} / \mathrm{h}$. Since these $R R$ s are more likely to occur in natural precipitation it is expected that the 3 -D effects will be more frequent at this higher frequency (even if with a little smaller amplitude than at the lower frequencies). 


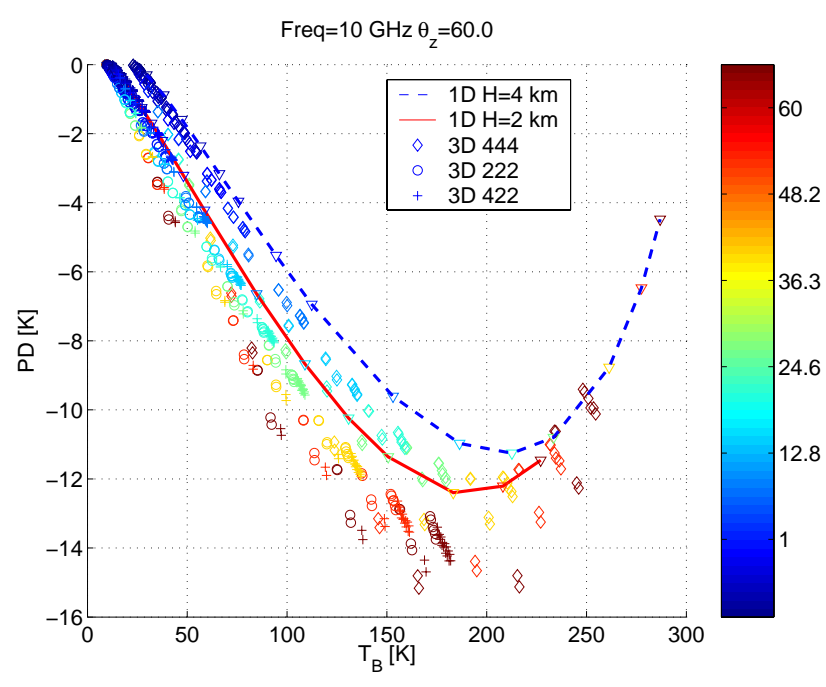

\section{Conclusions}

A simple box-type rain shaft has been selected to investigate 3 -D effects generated by rain-type dichroic media onto the signal sensed by upward looking ground-based polarimetric radiometers with channels around 10,19 and $30 \mathrm{GHz}$. Major findings can be summarized as following.

- Because of the reduction in the slant optical thickness for finite clouds, 3-D $T_{B}$ s assume intermediate values between clear sky and 1-D configuration values (left panels in Fig. 3). Well know leakages effects are confirmed as well.

- The polarization signal can strongly differ from its onedimensional counterpart (e.g. right panels in Fig. 3). When the observation point is located outside of the precipitating cloud, typical polarization patterns (with troughs and peaks) as a function of the observation angle are predicted.

- The most negative polarization differences are obtained at slant optical thicknesses around 1 (Fig. 4). Since in a 3-D configuration the same slant optical thickness is achieved at higher $R R \mathrm{~s}$ (which are characterized by more elongated raindrops) than in a 1-D configuration, 3-D box-type rain shaft produce stronger negative polarizations when the same slant optical thickness are sensed.

- A 1-D SP approximation is generally unsatisfactory when considering $T_{B} \mathrm{~s}$ and $P D \mathrm{~s}$ except at small $R R \mathrm{~s}$ and at the lower frequencies (Fig. 6). 3-D effects like inhomogeneous radiation field within the cloud (Fig. 7), $P D$ s different from zero at nadir (bottom right panel in Fig. 3) and non null third Stokes component (as high as 2 K, see Fig. 8) are peculiar of 3-D radiative transfer and cannot be accommodated by 1-D modeling.

- A wider variety of possibilities is achieved by 3-D modeling when producing scatter plots of $T_{B} \mathrm{~s}$ versus $P D \mathrm{~s}$ (Fig. 9). 3-D effects also allow to explain the time evolution of observations in the $\left(T_{B}, P D\right)$ plane also in terms of a shift of the rain shaft relative to the observation position, and not only in terms of a change either in the rain intensity or in the atmosphere vertical profile (like in 1-D modeling). 3-D effects can produce higher negative polarizations at $10 \mathrm{GHz}$ at very high $R R \mathrm{~s}$, at $19.4 \mathrm{GHz}$ at high $R R \mathrm{~s}$ and at $30.0 \mathrm{GHz}$ at intermediate $R R \mathrm{~s}(R R \leq 5 \mathrm{~mm} / \mathrm{h})$. Negative PD values as low as $-16 \mathrm{~K}$ are predicted by $3-\mathrm{D}$ scenarios (in accordance with observations). Due to the highest occurrence of low $R R$ s 3-D rain structure are believed to lower (increase), on average, the amplitude of the negative $P D$ signal at $10 \mathrm{GHz}(30 \mathrm{GHz})$.

9. Scatter plots of $T_{B} \mathrm{~s}$ versus $P D \mathrm{~s}$ at the three frequencies under investigation. A zenith angle $\theta_{z}=60^{\circ}$ is selected. The color bar modulates the rain rate of the rain shaft. See text for details.

- In retrieval algorithms which exploit the polarization signatures measured by ground-based radiometers 
(e.g. Czekala et al., 2001a) 3-D effects must be accounted for. To do this, besides the vertical temperature and water vapor profile of the atmosphere additional information about the horizontal structure of the clouds has to be collected. An ideal combination would consist in operating a polarimetric radiometer in synergy with a rain radar, able to capture the horizontal structure of the rain shaft; in addition otherwise, zenith scanning capabilities of the radiometer can be exploited.

- New measurements with scanning multifrequency polarimetric radiometers are highly recommended. Not only these measurements will be crucial for rain/cloud water discrimination but they will also provide a better insight and an independent confirmation of raindrop shapes and falling behavior modeling widely exploited by polarimetric radars.

Besides the 3-D effects here analysed, the discrimination technique between cloud and rain liquid water path based on ground-based polarimetry still requires dedicated studies. In particular, the impact of the drop size distribution assumption (in our study a Marshall and Palmer) onto the $T_{B}-P D$ relationship, the best selection of a set of frequencies to better face this uncertainty within the frame of a multi-spectral approach, and a precise quantification of the melting layer effect on $P D$ s remain open issues currently under study. To clarify these points, a project called ADMIRARI (ADvanced MIcrowave RAdiometer for Rain Identification) has been proposed by the authors at the German Science Foundation (now under final evaluation). If financed, it will provide a rich variety of measurements in rainy conditions from a three wavelength $(10,21$ and $37 \mathrm{GHz})$ polarized groundbased radiometer.

Acknowledgements. The authors are grateful to F. Evans for making the RT4 software available at the web-site http://nit.colorado.edu/polrad.html.

Edited by: W. E. Asher

\section{References}

Andsager, K., Beard, K. V., and Laird, N. S.: A laboratory study of oscillations and axis ratios for large raindrops, J. Atmos. Sci., 56, 2673-2683, 1999.

Battaglia, A. and Mantovani, S.: Forward Montecarlo Computations of Fully Polarized Microwave Radiation in Non Isotropic Media, J. Quant. Spectrosc. Radiat. Transfer, 95, 285-308, 2005.

Battaglia, A., Kummerow, C., Shin, D.-B., and Williams, C.: Toward characterizing the effect of radar bright bands on microwave brightness temperatures, J. Atmos. Ocean Technol., 20, 856-871, 2003.

Battaglia, A., Prodi, F., Porcu, F., and Shin, D.-B.: Measuring Precipitation from space: EURAINSAT and the future, chap. 3-D effects in MW radiative transport inside precipitating clouds: mod- eling and applications, edited by: Levizzani, V., Bauer, P., and Turk, F. J., Kluwer Academic edn., 2005.

Battaglia, A., Davis, C., Emde, C., and Simmer, C.: Microwave radiative transfer intercomparison study for 3-D dichroic media, J. Quant. Spectrosc. Radiat. Transfer, accepted, 2006.

Bauer, P., Schanz, L., and Roberti, L.: Correction of three dimensional effects for passive microwave remote sensing of convective clouds, J. Appl. Meteorol., 37, 1619-1632, 1998.

Bringi, V. N. and Chandrasekar, V.: Polarimetric Doppler Weather Radar, Principles and applications, Cambridge University Press, pp. 636, 2001.

Czekala, H.: Effects of ice particle shape and orientation on polarized microwave radiation for off-nadir problems, Geophys. Res. Lett., 25, 1669-1672, 1998.

Czekala, H. and Simmer, C.: Microwave radiative transfer with nonspherical precipitating hydrometeors, J. Quant. Spectrosc. Radiat. Transfer, 60, 365-374, 1998.

Czekala, H. and Simmer, C.: On precipitation induced polarization of microwave radiation measured from space, Meteorologische Zeitschrift, 11, 49-60, 2002.

Czekala, H., Havemann, S., Schmidt, K., Rother, T., and Simmer, C.: Comparison of microwave radiative transfer calculations obtained with three different approximations of hydrometeor shape, J. Quant. Spectrosc. Radiat. Transfer, 63, 545-558, 1999.

Czekala, H., Crewell, S., Hornbostel, A., Schroth, A., Simmer, C., and Thiele, A.: Interpretation of polarization features in ground based microwave observations as caused by horizontally aligned oblate rain drops, J. Appl. Meteorol., 40, 1918-1932, 2001a.

Czekala, H., Crewell, S., Simmer, C., and Thiele, A.: Discrimination of cloud and rain liquid water path by groundbased polarized microwave radiometry, Geophys. Res. Lett., 28, 267-270, 2001 b.

Davis, C. P., Emde, C., and Harwood, R. S.: A 3-D polarized reversed Monte Carlo radiative transfer model for $\mathrm{mm}$ and sub$\mathrm{mm}$ passive remote sensing in cloudy atmospheres, IEEE Trans. Geosci. Remote Sens., 43, 1096-1101, 2005.

Evans, K. F. and Stephens, G. L.: A new polarized atmospheric radiative transfer model, J. Quant. Spectrosc. Radiat. Transfer, 46, 413-423, 1991.

Evans, K. F. and Stephens, G. L.: Microwave radiative transfer through clouds composed of realistically shaped ice crystals. II. Remote sensing of ice clouds, J. Atmos. Sci., 52, 2058-2072, 1995.

Evans, K. F., Walter, S. J., Heymsfield, A. J., and Deeter, M. N.: Modeling of submillimiter passive remote sensing of cirrus clouds, J. Appl. Meteorol., 37, 184-205, 1998.

Haferman, J. L.: Light Scattering by Nonspherical Particles: Theory, Measurements, and Applications, chapter: Microwave scattering by precipitation, edited by: Mishchenko, M. I., Hovenier, J. W., and Travis, L. D., Academic Press, San Diego, 2000.

Haferman, J. L., Krajewski, W. F., Smith, T. F., and Sanchez, A.: Radiative transfer for a three-dimensional raining cloud, Appl. Opt., 32, 2795-2802, 1993.

Heymsfield, G. M. and Fulton, R.: Passive microwave and infrared structure of mesoscale convective systems, Meteorol. Atmos. Phys., 54, 123-139, 1994.

Janssen, M. A.: Atmospheric remote sensing by microwave radiometry, John Wiley \& Sons, 1996.

Kummerow, C. and Weinman, J. A.: Determining microwave brightness temperatures from precipitating horizontally finite and 
vertically structured clouds, J. Geophys. Res., 93, 3720-3728, 1988.

Kutuza, B. G., Zagorin, G. K., Hornbostel, A., and Schroth, A.: Physical modeling of passive polarimetric microwave observations of the atmosphere with respect to the third Stokes parameter, Radio Science, 33, 677-695, 1998.

Liu, Q. and Simmer, C.: Polarization and intensity in microwave radiative transfer, Beitr. Phys. Atmosph., 69, 535-545, 1996.

Liu, Q., Simmer, C., and Ruprecht, E.: Three-dimensional radiative transfer effects of clouds in the microwave spectral range, J. Geophys. Res., 101, 4289-4298, 1996.

Liu, Q.: Observation, Theory, and Modeling of Atmospheric Variability, chap. Polarimetric radiative transfer theory and its applications: An overview, edited by: Xun Zhu, World Scientific edn., pp 612, 2004.

Mätzler, C.: Thermal Microwave Radiation - Applications for Remote Sensing, IEE Electromagnetic Waves Series, London, UK, 2006.

Mishchenko, M. I.: Calculation of the amplitude matrix for a nonspherical particle in a fixed orientation, Appl. Opt., 39, 10261031, 2000.

Prigent, C., Pardo, J., Mishchenko, M. I., and Rossow, W. B.: Microwave polarized scattering signatures in clouds: SSM/I observations interpreted with radiative transfer simulations, J. Geophys. Res., 106, 28 243-28 258, 2001.

Prigent, C., Defer, E., Pardo, J., Pearl, C., Rossow, W. B., and Pinty, J.-P.: Relations of polarized scattering signatures observed by the TRMM Microwave Instrument with electrical processes in cloud systems, Geophys. Res. Lett., 32, L04810, doi:10.1029/2004GL022225, 2005.
Raynaud, L., Chenerie, I., and Lemorton, J.: Modeling of radiowave scattering in the melting layer of precipitation, IEEE Trans. Geo. Rem. Sens., 38, 1574-1584, doi:10.1109/36.851957, 2000.

Roberti, L. and Kummerow, C.: Monte Carlo calculations of polarized microwave radiation emerging from cloud structures, J. Geophys. Res., 104, 2093-2104, 1999.

Roberti, L., Haferman, J., and Kummerow, C.: Microwave radiative transfer through horizontally inhomogeneous precipitating clouds, J. Geophys. Res., 99, 707-716, 1994.

Spencer, R. W., Goodman, H. M., and Hood, R. E.: Precipitation retrieval over land and ocean with the SSM/I: identification and characteristics of the scattering signal, J. Atmos. Ocean Technol., 6, 254-273, 1989.

Troitsky, A. V., Osharin, A. M., Korolev, A. V., and Strapp, J. W.: Polarization of Thermal Microwave Atmospheric Radiation due to Scattering by Ice Particles in Clouds, J. Atmos. Sci., 60, 1608$1620,2003$.

Weinman, J. A. and Davies, R.: Thermal microwave radiances from horizontally finite clouds of hydrometeors, J. Geophys. Res., 83, 3099-3107, 1978.

Zhang, W., Tervonen, J., and Salonen, E.: Backward and forward scattering by the melting layer composed of spheroidal hydrometeors at 5-100 GHz, IEEE Transactions on Antennas and Propagation, 44, 1208-1219, doi:10.1109/8.535378, 1996. 